\title{
Modeling Update for the Thirty Meter Telescope Laser Guide Star Dual-Conjugate Adaptive Optics System
}

\author{
Luc Gilles $^{a}$, Lianqi Wang ${ }^{a}$ and Brent Ellerbroek ${ }^{a}$ \\ ${ }^{a}$ Thirty Meter Telescope Project Office \\ 1200 E. California Boulevard, Mail Code 102-8 Pasadena, CA 91125
}

\begin{abstract}
This paper describes the modeling efforts undertaken in the past couple of years to derive wavefront error (WFE) performance estimates for the Narrow Field Infrared Adaptive Optics System (NFIRAOS), which is the facility laser guide star (LGS) dual-conjugate adaptive optics (AO) system for the Thirty Meter Telescope (TMT). The estimates describe the expected performance of NFIRAOS as a function of seeing on Mauna Kea, zenith angle, and galactic latitude (GL). They have been developed through a combination of integrated AO simulations, side analyses, allocations, lab and lidar experiments.
\end{abstract}

Keywords: Thirty Meter Telescope, Laser guide stars, Modeling, Adaptive Optics

\section{INTRODUCTION}

NFIRAOS (Narrow Field Infrared Adaptive Optics System) is the dual-conjugate, laser guide star (LGS), facility adaptive optics (AO) system for the Thirty Meter Telescope (TMT) [1,2]. Its top-level requirements as specified in the Observatory Requirements Document (ORD) include:

1. $85 \%$ throughput from 0.8 to $2.5 \mu \mathrm{m}$.

2. Thermal emission below $15 \%$ of the black body radiation from the telescope and atmosphere at ambient temperature.

3. 191nm root-mean-square (RMS) wavefront error (WFE) over the IRIS client instrument [3] 17" $\times 17$ " field of view (FoV) in imaging mode at zenith under median turbulence conditions.

4. $30 \%$ enclosed energy in J band in the IRMS client instrument [4] 80mas slits over a circular 2' diameter FoV.

5. $50 \%$ sky coverage (sc) at the galactic pole (GP) with at most 2 mas rms Tip/Tilt jitter at zenith under median turbulence conditions.

6. $2 \%$ differential photometry for a 10 min exposure on a $30 "$ FoV at $1 \mu \mathrm{m}$.

7. $50 \mu$ as differential astrometry for a $100 \mathrm{~s}$ exposure on a $30 " \mathrm{FoV}$ in $\mathrm{H}$ band.

The fundamental design parameters derived from these top-level requirements include:

1. 6 LGS Shack-Hartmann (SH) wavefront sensors (WFSs) of order $60 \times 60$ observing a 70 " diameter asterism (5 LGSs on a circle +1 on-axis); polar coordinate detectors with $\sim 205,000$ pixels/WFS resulting in $\sim$ 5,800 gradients/WFS.

2. two piezo stack deformable mirrors (DMs) conjugate to 0 and $11.2 \mathrm{~km}$ range, respectively of $63 \times 63$ and $76 \times 76$ actuators with $5 \mathrm{~mm}$ pitch; ground DM mounted on a Tip/Tilt stage (TTS).

3. real-time controller solving a $\sim 35,000 \times 7,000$ wavefront control problem at $800 \mathrm{~Hz}$ with an end-to-end latency of $1 \mathrm{~ms}$.

Send correspondence to lgilles@caltech.edu

Adaptive Optics Systems II, edited by Brent L. Ellerbroek, Michael Hart, Norbert Hubin, Peter L. Wizinowich, Proc. of SPIE Vol. 7736, 77360W - @ 2010 SPIE · CCC code: 0277-786X/10/\$18 - doi: 10.1117/12.858279 
4. measurements from on-instrument natural guide star (NGS) SH-WFSs (OIWFSs): 2 Tip/Tilt (TT) and 1 Tip/Tilt/Focus/Astigmatism (TTFA) OIWFSs sensing in the near-infrared at 10-800 Hz and patrolling an unvignetted 2' field.

Note that up to date, the NFIRAOS system requirements have only been defined at zenith, with NGS densities for the GP, and will be updated in the near-future for more general operating conditions. Our off-zenith performance estimates will be compared in this paper against a naive $\sqrt{\sec (\psi)}$ air-mass based extrapolation of the requirement at zenith, where $\psi$ denotes zenith angle.

Performance analysis is a critical task providing a comprehensive (i) evaluation of the overall AO architecture, and (ii) verification that the system meets all its requirements. Performance analysis should therefore capture all the effects of all sources of wavefront disturbances (atmosphere, telescope and AO system with all its processing algorithms). Performance evaluation has been carried out as a function of seeing on Mauna Kea, zenith angle and galactic latitude (GL). Estimates were developed through a combination of integrated AO simulations, side analyses, allocations, lab and lidar experiments. Our main results are that:

1. The $191 \mathrm{~nm}$ requirement over a $17 " \times 17 "$ FoV at zenith with $50 \%$ sc at the GP, under median turbulence conditions is met with $\sim 85 \mathrm{~nm}$ RMS contingency.

2. The extrapolated requirement of $205 \mathrm{~nm}$ at $\psi=30^{\circ}$ at $50 \% \mathrm{sc}$ at the GP is met with $\sim 69 \mathrm{~nm}$ RMS contingency.

3. The extrapolated requirement of $227 \mathrm{~nm}$ at $\psi=45^{\circ}$ at $50 \% \mathrm{sc}$ at the GP is not met by a significant amount, nonetheless:

(a) $227 \mathrm{~nm}$ at $\psi=45^{\circ}$ is met at $30 \% \mathrm{sc}$ at the GP with $\sim 55 \mathrm{~nm}$ RMS contingency.

(b) $227 \mathrm{~nm}$ at $\psi=45^{\circ}$ is met at $50 \% \mathrm{sc}$ at $30^{\circ}$ GL with $\sim 83 \mathrm{~nm}$ RMS contingency.

Full scientific assessment of off-zenith performance versus sky coverage is underway, but preliminary analysis on a set of 75 specific target fields covering 5 different field types (extragalactic, nearby galaxies, central black holes, massive young star clusters, and yellow super giants) indicate that only a few percent of these targets are not expected to meet the extrapolated ORD WFE requirement at their culminating elevation angle (minimum zenith angle) with $50 \%$ or better probability under median Mauna Kea turbulence conditions.

Table 1 shows the top-level error terms of the performance estimates at zenith. WFE has been decomponsed into mutually orthogonal LGS and NGS modes, which are the modes controlled in the baseline "split tomography" control architecture of NFIRAOS [5]. The NGS modes consists of TT and 3 fixed "plate scale modes" that induce only TT in the LGS WFSs (unobservable), and field-dependent TT plus quadratic WFE in the OIWFSs and science directions. To first-order, the LGS loop is thus decoupled from the NGS asterism and NGS modes, enabling efficient sky coverage analysis via post-processing [6]. An overview of the simulation tools for performance analysis is provided in Section 2, followed in Sections 3 to 6 by a description of the breakdown of each of the top-level terms.

\begin{tabular}{|l|l|}
\hline Zenith angle $(\psi$, deg $)$ & 0 \\
\hline DM Fitting FoV diameter & $30 "$ circular \\
\hline Eval. FoV & $17^{\prime \prime} \times 17^{\prime \prime}$ \\
\hline ORD & $\mathbf{1 9 1}$ \\
\hline LGS mode & \multicolumn{2}{|c|}{160} \\
\hline Fundamental error terms & 127 \\
\hline Opto-mechanics & 74 \\
\hline AO components and higher-order effects & 63 \\
\hline NGS mode @ 50\% sc @ GP & 61 \\
\hline Contingency & 85 \\
\hline
\end{tabular}

Table 1. Top-level terms of the NFIRAOS performance estimates for median Mauna Kea seeing at zenith. 


\section{SIMULATION TOOLS FOR PERFORMANCE ANALYSIS}

As mentioned in the Introduction, NFIRAOS will use a "split tomography" control architecture with separate processing of the LGS and NGS WFS measurements in such a way that the LGS component of the tomographic estimation does not depend upon the NGS asterism (location and brightness). The main benefits of such an approach are: (i) a simpler formulation of minimum variance atmospheric tomography allowing for algorithms with reduced computational complexity and cost (processing requirements), and (ii) a separate, flexible control of a small set of NGS-controlled modes that can operate at a lower frame rate than the LGS loop depending upon the brightness of the NGSs. So far, a baseline (ad hoc) split similar to the one developed a decade ago for the MCAO system of the Gemini South telescope [7] has been studied in detail [5]. In such a formulation, the NGS modes consist of 5 fixed modes defined on 2 DMs at different conjugate ranges: TT and 3 "plate scale modes" (quadratic modes of opposite signs and scaled by the LGS cone compression factor). Such a simple definition of the NGS modes lead to the following useful properties: (i) they induce only TT in the LGS WFSs (hence to first-order, the LGS loop is decoupled from the NGS loop), and (ii) they induce field-dependent TT plus fieldindependent quadratic wavefront aberrations resulting from the LGS cone effect in the NGS WFSs and science directions (which needs to be corrected by the NGS loop to avoid image distortion). The fact that to first-order the LGS loop is decoupled from the NGS loop, enables efficient statistical sky coverage analysis over hundreds of asterisms in post-processing [6]. A block diagram illustrating this concept is provided in Fig.1 (without temporal filtering for simplicity). The NGS control loop uses a noise-weighted rank 5 modal least-squares reconstructor,

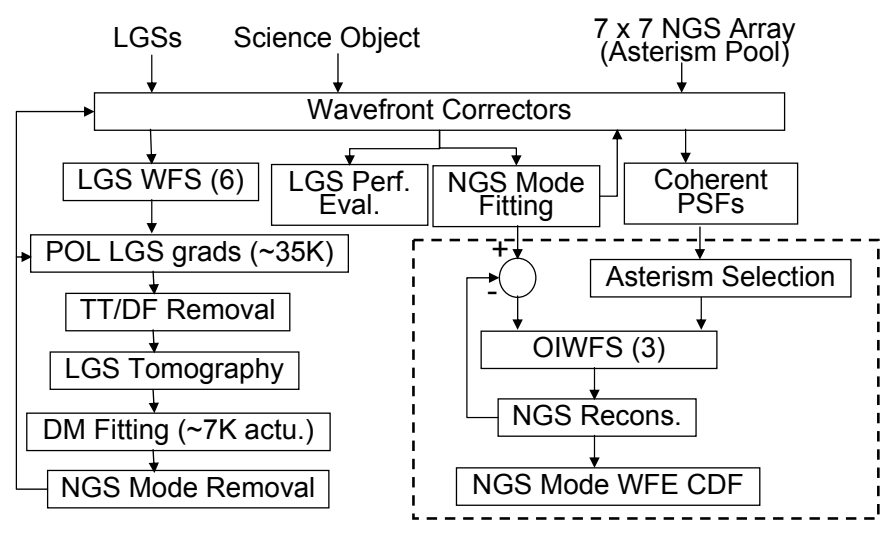

Sky coverage Post-processor

Figure 1. NFIRAOS baseline split tomography control architecture allowing efficient statistical sky coverage analysis via post-processing.

and the coupling of the LGS loop into the NGS loop is further reduced by nulling the component of the LGScontrolled DM commands that lies in the span of the NGS-controlled modes. This component is nonzero on account of LGS WFS measurement noise and the use of a minimum variance estimator (nonzero statistical coupling between TT and coma).

Performance evaluation of the LGS and NGS control loops follows a 2-step process. In the first step, an endto-end high-order simulation of the LGS control loop is performed, during which the 5 NGS modes contained in the atmosphere are corrected perfectly (least-squares fit) without the degrading effects of WFS aliasing, noise, physical optics effects and servo lag. At every time step the following information is saved:

1. 5 coherent (complex-valued) point spread functions (PSFs) (4 over a $2 \times 2$ array of subapertures and 1 over the full-aperture) is recorded for 29 NGS locations (asterism pool) partitioning the NFIRAOS 2' patrol FoV on a grid of 20 " sampling. In order to reduce storage requirements, only the central $32 \times 32$ portion of theses PSFs is stored.

2. the ideal coefficients for the NGS modes. 
During the post-processing step, for a given random guide star field (realization of a 2' field obtained from the Besancon guidestar model) containing 2 TT and 1 TTFA stars pre-selected as best asterism based on a firstorder geometrical performance analysis (evaluation of all combinations of stars is computationally impractical), the actual NGS mode coefficient time history can be estimated from the ideal coherent PSFs and ideal NGS mode coefficients. This is done by applying an inverse Fourier transform to each coherent PSF and multiplying the result by the complex exponential of the differential wavefront induced by the ideal and actual NGS mode coefficients. This process is initialized with identical actual and ideal NGS mode coefficients, and is repeated for several different NGS sampling frequencies. At each sampling frequency, NGS modal gains are computed to balance the error rejection against noise propagation [6]. A type II controller is used for all 5 NGS modes. Results are sorted typically over 500 realizations of NFIRAOS guide star fields, and a cumulative distribution function (CDF) of the NGS mode WFE is finally obtained.

The high-order simulation of the LGS control loop is performed in a recently developed multi-threaded simulator called MAOS. The code is written in ANSI C, and uses the blas, lapack and fftw libraries. It runs on a linux operating system, and incorporates a built-in job scheduler and a data vizualization tool. On a dual quad-core Intel Xeon 3.3GHz (Nehalem architecture), a typical NFIRAOS simulation takes from 8-2 sec per time step depending on the number of threads used; the highest speed is reached at 6 threads (number of LGS WFSs). We are thus now in a position to simulate 1 min of telescope time in about 24 hours, allowing us to study background LGS processes like centroiding algorithm update, focus tracking, etc. Typical memory requirements are on the order of $2 \mathrm{~GB}$ for a simulation with $74096 \times 4096$ atmospheric screens.

A block diagram illustrating how the wavefront error budget is organized is shown in Fig.2.

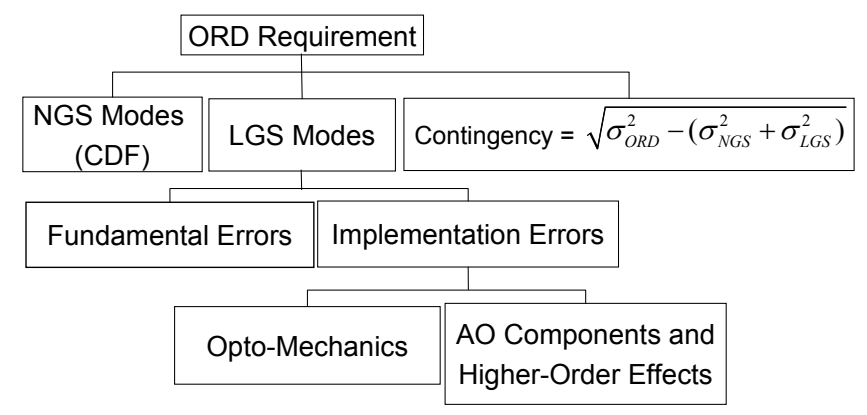

Figure 2. Organization of the NFIRAOS wavefront error budget.

All our performance analysis has been done for 25\%, $50 \%$ and $75 \%$ Mauna Kea seeing. These profiles were obtained by first computing the combined wavefront error due to DM fitting and servo lag for each Mauna Kea turbulence profile measurement collected by the TMT Site Testing Group, and then averaging all of the profiles with wavefront errors that fall within a $\pm 5 \%$ band around the $25 \%, 50 \%$ and $75 \%$ values of this quantity. Relevant turbulence and wind profile parameters are summarized in Fig.3(a) and 3(b). The wind profile was obtained by down-sampling (using the MASS triangular influence functions) high-resolution night-time wind profiles obtained from the NCEP/NCAR (National Center for Environmental Prediction/Atmospheric Research) reanalysis project $[8]$.

\section{BREAKDOWN OF FUNDAMENTAL ERROR TERMS}

Each fundamental error term can be evaluated separately via simulations once an ordering has been decided upon, and simulations are run by cumulatively including error terms in that order. The budget is then derived by quadrature differences. Table 2 shows our chosen ordering and the value of each term for the median Mauna Kea turbulence profile of Fig.3(a), 3(b). The first 6 terms were evaluated over an annular aperture of outer diameter equal to $30 \mathrm{~m}$ and inner diameter equal to $3.6 \mathrm{~m}$ ( $12 \%$ central obscuration). Direct Cholesky factorization of the tomography and fitting matrices was used for the first 7 terms. 


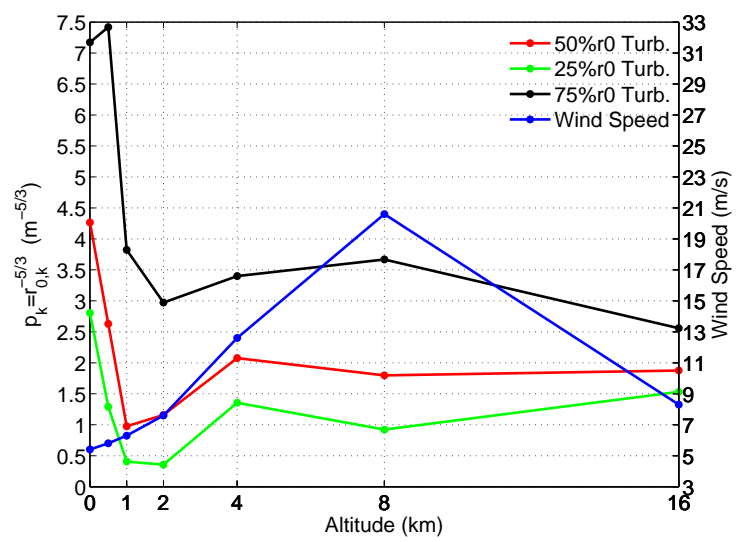

(a)

\begin{tabular}{|l|l|l|l|l|l|l|l|}
\hline Altitude $(\mathrm{km})$ & 0 & 0.5 & 1 & 2 & 4 & 8 & 18 \\
\hline Wind Speed $(\mathrm{m} / \mathrm{s})$ & 5.6 & 5.8 & 6.2 & 7.6 & 13 & 19 & 12 \\
\hline $25 \%$ seeing, $r_{0}=27.4 \mathrm{~cm}, \theta_{0}=2.7 ", \theta_{2}=10.2 ", f_{G}=15.9 \mathrm{~Hz}$ \\
\hline Weights $(\%)$ & 32 & 15 & 4.7 & 4.1 & 16 & 11 & 18 \\
\hline $50 \%$ seeing, $r_{0}=19.9 \mathrm{~cm}, \theta_{0}=2.2^{\prime \prime}, \theta_{2}=8.2 ", f_{G}=21.7 \mathrm{~Hz}$ \\
\hline Weights $(\%)$ & 29 & 18 & 6.6 & 7.8 & 14 & 12 & 13 \\
\hline $75 \%$ seeing, $r_{0}=12.7 \mathrm{~cm}, \theta_{0}=1.7^{\prime \prime}, \theta_{2}=6 ", f_{G}=32 \mathrm{~Hz}$ \\
\hline Weights $(\%)$ & 23 & 24 & 12.3 & 9.6 & 11 & 11.8 & 8.3 \\
\hline
\end{tabular}

(b)

Figure 3. (a) Mauna Kea turbulence and wind profiles. (b) Mauna Kea turbulence and wind profile parameters.

\begin{tabular}{|l|l|}
\hline Zenith angle $(\psi$, deg) & 0 \\
\hline DM Fitting FoV diameter & $30^{\prime \prime}$ circular \\
\hline Eval. FoV & $17^{\prime \prime} \times 17^{\prime \prime}$ \\
\hline Tot. Fundamental WFE (nm) & $\mathbf{1 2 7}$ \\
\hline DM Fitting & 63 \\
\hline DM Projection & 66 \\
\hline Tomography+WFS aliasing & 50 \\
\hline Servo lag & 31 \\
\hline Physical optics WFS & 15 \\
\hline WFS noise & 41 \\
\hline TMT pupil function & 5 \\
\hline RTC solvers (tomo/fitting) & 10 \\
\hline simulation screen undersampling & 26 \\
\hline Cn2 profile undersampling & 40 \\
\hline
\end{tabular}

Table 2. Breakdown of fundamental error terms for median Mauna Kea seeing at zenith.

A brief description of each error term is provided below.

1. The DM fitting error is defined as the RMS difference between a wavefront and its least-squares fit (projection) onto the span of the influence functions of all DMs. The least-squares projection is restricted to a single-direction (on-axis fitting field), and performance is evaluated in that direction (on-axis performance evaluation field). This error is driven by the inter-actuator spacing and actuator geometry on the DMs. The DM least-squares fit has been computed for a down-sampled atmosphere ( $1 / 4 \mathrm{~m}$ resolution grids), and bicubic DM influence functions with $30 \%$ inter-actuator coupling and $1 / 2 \mathrm{~m}$ inter-actuator pitch $(63 \times 63$ ground conjugated DM, and $76 \times 76$ altitude conjugated DM). The DM fitting error is significantly better than would be obtained with a single DM on account of the interlaced DM geometry of NFIRAOS.

2. The DM projection error is defined as the incremental (in quadrature) fitting error for DM actuators commanded to minimize the field-averaged RMS WFE for wavefronts across an extended FoV. RMS WFE may then be evaluated for one or several directions defining the performance evaluation field, which is not necessarily identical with the DM fitting field. This error is driven by the number and range of the DMs, and has been computed for the NFIRAOS DM conjugate ranges of 0 and $11.2 \mathrm{~km}$ (selected by trade study). 
3. The combined tomography and WFS spatial aliasing error is defined as the incremental (in quadrature) error arising from the use of multiple WFSs modeled as noise-free average gradient sensors operating in open-loop and a minimum variance wavefront reconstructor. This error is driven by the order of the WFSs, and guide star asterism geometry. The error has been computed for the NFIRAOS order $60 \times 60$ LGS WFSs and asterism (5 LGSs on a 70" diameter circle + 1 LGS on-axis). A $60 \%$ subaperture illumination threshold has been used, which provides optimal performance.

4. The servo lag (or bandwidth) error is defined as the incremental (in quadrature) error arising in closedloop. This error is driven by the temporal sampling frequency $(800 \mathrm{~Hz})$ and processing latency of the loop (standard integrator with 2 frames of latency and a gain of $1 / 2$ ).

5. The error related to the use of a physical optics WFS model is defined as the incremental (in quadrature) error arising from the use of a physical optics model for the WFSs with bright point sources. The error has been computed for the polar coordinate detector [9] and a constrained matched filter centroiding algorithm [10].

6. The WFS measurement noise is defined as the incremental error arising from measurement noise. This error is driven by the guide star signal level (photon noise), the spot size, the WFS detector parameters (read noise) and the centroiding algorithm (noise propagation). The error has been evaluated for a signal level of 900 photo-detected electrons (PDEs) per subaperture per frame at $800 \mathrm{~Hz}$, and 3 electrons readout noise per pixel per frame. With D2a/D2b pumping and circular polarization, such a signal level is expected to be met at Mauna Kea with a $25 \mathrm{~W}$ laser beam/LGS at a pessimistic low sodium column density of $1.9 \times 10^{13}$ atoms $/ \mathrm{m}^{2}$.

7. The error induced by the TMT pupil function is defined as the incremental error arising from the telescope primary mirrror (M1) segment gaps and obscurations due to the secondary mirror (M2) support trusses and cables.

8. The error induced by the RTC algorithms is defined as the incremental error arising from the use of iterative algorithms to provide an approximate solution to the tomography and DM fitting matrix systems. This error has been evaluated for the conjugate gradient (CG) algorithm with 30 iterations for the tomography step, and 4 iterations for the DM fitting step.

9. The simulation undersampling error is defined as the incremental (in quadrature) spatial aliasing error which is unsampled with discrete turbulence phase screens $(1 / 64 \mathrm{~m}$ resolution). The error has been estimated by fitting $\sigma^{2}(a, b, c)=c\left(1-a(\Delta x)^{b}\right)$, where $a, b, c$ are model parameters, to simulation results $\sigma_{\text {sim }}^{2}(\Delta x)$ obtained with $\Delta x=1 / 16,1 / 32,1 / 64,1 / 128,1 / 256 \mathrm{~m}$. The error is then $\sqrt{c-\sigma_{\text {sim }}^{2}(\Delta x=1 / 64)}$.

10. The $\mathrm{Cn} 2$ profile undersampling error is defined as the incremental (in quadrature) aliasing error arising from the use of a small number (7) of simulated atmospheric layers. The error has been estimated by running simulations with 25 layers simulating the atmosphere, while keeping the number of reconstructed layers fixed at 6 .

\section{OPTO-MECHANICAL IMPLEMENTATION ERRORS}

A breakdown of the opto-mechanical implementation errors is shown in Table 3. 


\begin{tabular}{|c|c|}
\hline Total (nm) & \multirow[t]{2}{*}{74} \\
\hline Telescope pupil misregistration & \\
\hline Telescope/Observatory wavefront errors & 41 \\
\hline Static M1 errors & \multirow{6}{*}{$\begin{array}{l}31 \\
10 \\
14 \\
16 \\
14\end{array}$} \\
\hline Static M2/M3 errors & \\
\hline Dynamic M1 segments & \\
\hline Dome seeing & \\
\hline Mirror seeing & \\
\hline NFIRAOS & \\
\hline Uncorrectable errors & \multirow{5}{*}{$\begin{array}{l}35 \\
35 \\
14 \\
16\end{array}$} \\
\hline NCPA calibration errors & \\
\hline DM-to-WFS pupil distortion & \\
\hline DM-to-WFS pupil misregistration & \\
\hline Science Instrument & \\
\hline
\end{tabular}

Table 3. Breakdown of the opto-mechanical implementation error terms for median Mauna Kea seeing at zenith.

A brief description of each error term is provided below.

1. Telescope pupil misregistration was simulated for a single-conjugate AO system by misregistering in translation a single on-axis LGS WFS and a single DM by a common amount equal to $0.3 \%$ of the pupil diameter, which is the ORD requirement on input pupil misregistration.

2. Evaluation of the static M1/M2/M3 errors was performed from representative OPD maps that included segment passive support errors, segment piston/tip/tilt errors, gravity clocking and decenter errors, segment figuring error with warping harness correction and thermal disturbances.

3. Regarding the evaluation of the dynamic M1 errors, 32 different OPD realizations of wind-driven M1 segment misalignments induced by a $1.5 \mathrm{~m} / \mathrm{s}$ wind speed at M1 were simulated. The residual error was found approximately equal to $14 \mathrm{~nm}$ for a $1 \mathrm{~Hz}$ control banwidth of the primary mirror control system (M1CS) (input disturbance was 45nm RMS).

4. Dome seeing and mirror seeing are currently allocations.

5. Uncorrectable errors are mirror polishing and gravitational/thermal print-through errors of spatial frequencies greater than 1 cycle/m (DM cutoff frequency).

6. Non-common-path aberrations (NCPA) are assumed to be $80 \%$ correctable by calibration (35 nm residual of $175 \mathrm{~nm}$ errors).

7. DM-to-WFS pupil distortion in the LGS WFS optical design was obtained from a 5th order polynomial fit of distorted projected lenslet coordinates onto the ground level DM. The residual error was found on the order of $14 \mathrm{~nm}$ for a $100 \mathrm{~km}$ LGS focus range.

8. DM-to-WFS pupil translational and rotational misregistration was obtained for misregistrations of magnitude equal to $10 \%$ of a subaperture.

9. Science instrument. A 30nm allocation has been budgeted for this term.

\section{AO COMPONENT ERRORS AND HIGHER-ORDER EFFECTS}

A breakdown of the AO component errors and higher-order effects is shown in Table 4. 


\begin{tabular}{|c|c|}
\hline Total (nm) & 63 \\
\hline DM & 49 \\
\hline Saturation & 0 \\
\hline Hysteresis & 19 \\
\hline Influence function & 0 \\
\hline Flattening & 45 \\
\hline LGS WFS and Na layer & 34 \\
\hline Stale matched filter (offset/gain calibration) & 14 \\
\hline Na layer range tracking & 12 \\
\hline Rayleigh backscattering & 1 \\
\hline Momentary signal level variation & 23 \\
\hline Differential atmospheric refraction & 16 \\
\hline Control algorithm numerical precision & 21 \\
\hline
\end{tabular}

Table 4. Breakdown of the AO component errors and higher-order effects for median Mauna Kea seeing at zenith.

A brief description of each error term is provided below.

1. The NFIRAOS DM stroke requirement is $10 \mu \mathrm{m}$ peak-to-valley for both DMs, which is sufficient to correct up to $5 \times$ the piston/tip/tilt removed wavefront error at the edge of the aperture for a von Karman turbulence profile with a $10 \mathrm{~cm}$ Fried parameter and a $60 \mathrm{~m}$ outer scale. $2 \mu \mathrm{m}$ additional stroke is available for telescope, AO and instrument errors. Saturation effectively never occurs in simulations.

2. The hysteresis budget was derived from simulations with $5 \%$ hysteresis as measured by CILAS on their TMT subscale DM demo.

3. The DM influence function is modeled as a bi-cubic spline with $30 \%$ inter-actuator coupling, which provides an excellent fit to the measured influence function of the subscale demo.

4. The DM flattening error is an allocation based upon measured performance of the subscale demo at an operating temperature of $-35^{\circ} \mathrm{C}$.

5. Regarding the LGS WFS and sodium layer error terms, a conservative 10nm allocation has been budgeted for offset calibration, based on a $0.3 \mathrm{~Hz}$ update rate provided by a moderate order (MOR) truth WFS. A similar $10 \mathrm{~nm}$ allocation has been budgeted for gain calibration (delay), based on a $0.3 \mathrm{~Hz}$ update rate provided by line-of-sight dithering. These updates will lag the actual variations in the sodium profile, which will induce a reconstruction error concentrated in low-order Zernike modes.

6. Sodium layer range variability also induces a focus error in the LGS WFS measurements, therefore causing an equal focus error in the tomographic wavefront reconstruction. This error will be measured and corrected by the TTFA NGS WFS. The residual focus error was computed analytically using the power spectral density (PSD) of the sodium layer range varations and the rejection transfer function of the focus tracking loop for a TTFA sampling rate of $90 \mathrm{~Hz}$ [11].

7. Rayleigh backscattering has been modeled in detail [12] and was found neglegible for NFIRAOS due to the very small fraction of subapertures affected ( $0.4 \%$ at zenith). The effect has been estimated on the order of $12 \mathrm{~nm}$ without calibration, and on the order of $1 \mathrm{~nm}$ with $80 \%$ calibration. Matched filter updates at $0.3 \mathrm{~Hz}$ are expected to provide the required calibration accuracy to better than $80 \%$.

8. Momentary signal level variations are due to ozone, aerosol, cirrus scattering and laser power fluctuations occuring on time scales shorted than $0.3 \mathrm{~Hz}$ (and are therefore not taken into account in the matched filters); the corresponding error was estimated on the order of $23 \mathrm{~nm}$ for a $20 \%$ reduction.

9. Differential atmospheric refraction arises from the fact that the AO system will slightly over-correct atmospheric turbulence since the refractive index (and hence the OPD) at $589 \mathrm{~nm}$ is $1.3 \%$ larger than in J band. The resulting error is about $16 \mathrm{~nm}$ for a turbulence outer scale of $30 \mathrm{~m}$. 
10. Control algorithm numerical precision refers to the error induced by finite precision arithmetic (4 bytes).

\section{SKY COVERAGE ANALYSIS RESULTS}

As described in Sec.2, statistical sky coverage is performed in post-processing over hundreds of guide star fields. The performance characteristics of the H2RG OIWFS detector has been modeled in detail, and the matched filter pixel processing algorithms and type II woofer-tweeter control law have been tuned to optimize performance. The ORD specifies that the atmospheric and telescope TT errors need to be corrected to a residual of 2 mas RMS (2-axis, i.e. $\sim 72 \mathrm{~nm}$ RMS WFE) at $50 \%$ sky coverage at the galactic pole (GP). Simulation results indicate that this requirement is met with margin at zenith, and that off-zenith performance is limited by physical optics effects. As illustrated in Fig.4(a) and 4(b), lower NGS Strehls across the 2' patrol field, together with smaller isoplanatic and generalized isoplanatic angles, $\theta_{0}$ and $\theta_{2}$, gradually lead to the loss of diffraction-limited PSF cores at large offsets in the patrol field. This complicates the selection of preferred asterisms for each simulated guide star field, since a faster geometrical model has been used for this purpose. This effect has been unobserved previously with geometrical OIWFS models excluding physical optics effects. Performance is improved by limiting the OIWFS patrol FoV to $\sim 1.3$ ' diameter at 30 degree zenith angle and to $\sim$ 0.7' diameter at 45-60 degree. The CDF of the total NGS mode WFE at the galactic pole obtained with such a patrol FoV reduction is shown in Fig.4(c) for various zenith angles. Preliminary simulation results implementing "ideal" MOAO correction behind NFIRAOS are displayed in Fig.4(d), and illustrate that such a correction provides diffraction-limited PSF cores at all zenith angles.

Finally, the grand total NFIRAOS performance estimates at $\psi=0,30,45$ deg is expressed in Table 5 as a contingency against the $\sqrt{\sec (\psi)}$ extrapolated ORD requirement at zenith (a negative value means that the estimate exceeds the extrapolated requirement).

\begin{tabular}{|c|c|c|c|}
\hline Zenith angle $(\psi$, deg $)$ & 0 & 30 & 45 \\
\hline DM Fitting FoV diameter & \multicolumn{3}{|c|}{$30 "$ circular } \\
\hline Eval. FoV & \multicolumn{3}{|c|}{$17^{\prime \prime} \times 17^{\prime \prime}$} \\
\hline $\mathbf{O R D} \star \sqrt{\sec (\psi)}$ & 191 & 205 & 227 \\
\hline Contingency@50\% sc @ GP, 50\%r0 & 85 & 69 & -185 \\
\hline Contingency @ 30\% sc @ GP, 50\%r0 & 94 & 94 & 55 \\
\hline Contingency@ 10\% sc @ GP, 50\%r0 & 100 & 104 & 97 \\
\hline Contingency@ $50 \%$ sc @ 30 GL, 50\%r0 & 100 & 105 & 83 \\
\hline Contingency@30\% sc @ 30 GL, 50\%r0 & 103 & 107 & 97 \\
\hline Contingency@10\% sc @ 30 GL, 50\%r0 & 104 & 109 & 106 \\
\hline Contingency@ $90 \%$ sc @ GP, 25\%r0 & 116 & 118 & -27 \\
\hline Contingency@30\% sc @ GP, 25\%r0 & 120 & 128 & 120 \\
\hline Contingency@10\% sc @ GP, 25\%r0 & 123 & 133 & 137 \\
\hline
\end{tabular}

Table 5. Grand total NFIRAOS performance estimates expressed as a contingency against the $\sqrt{\sec (\psi)}$ extrapolated ORD requirement at zenith.

\section{CONCLUSION}

Extensive performance analysis for the NFIRAOS system indicates that:

1. The $191 \mathrm{~nm}$ requirement at zenith at $50 \% \mathrm{sc}$ at the GP under median turbulence conditions over a $17 " \times$ 17 " FoV is met with $\sim 85 \mathrm{~nm}$ RMS contingency.

2. The extrapolated requirement of $205 \mathrm{~nm}$ at $\psi=30^{\circ}$ at $50 \% \mathrm{sc}$ at the GP is met with $\sim 69 \mathrm{~nm}$ RMS contingency. 


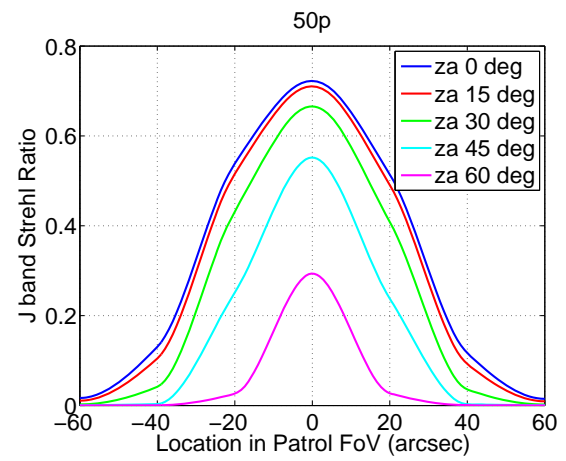

(a)

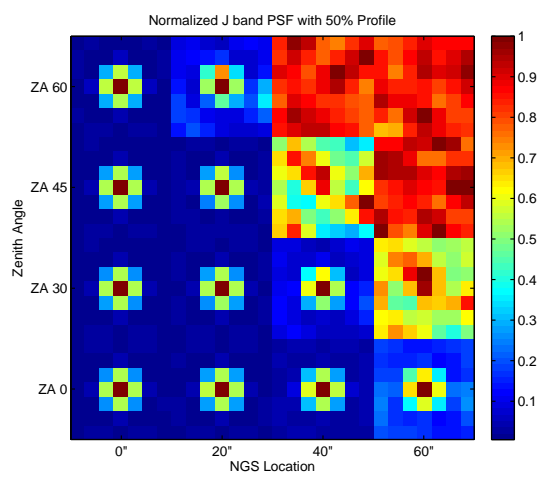

(b)

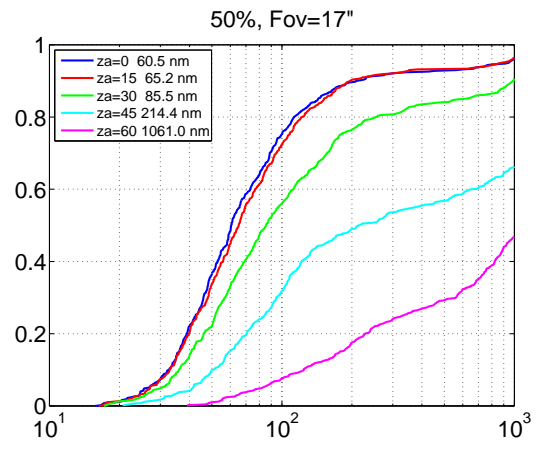

(c)

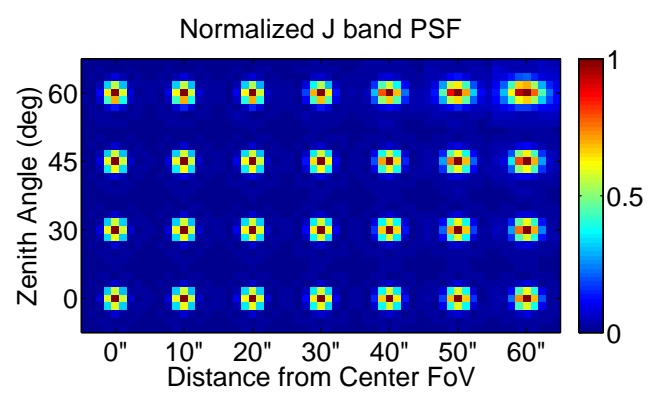

(d)

Figure 4. (a) J band Strehl ratio versus zenith angle. (b) Normalized J band PSF versus zenith angle and NGS location. (c) Total NGS mode CDF at the galactic pole versus zenith angle. (d) Normalized J band PSF obtained with ideal MOAO correction behind NFIRAOS.

3. The extrapolated requirement of $227 \mathrm{~nm}$ at $\psi=45^{\circ}$ at $50 \% \mathrm{sc}$ at the GP is not met by a significant amount, nonetheless:

(a) $227 \mathrm{~nm}$ at $\psi=45^{\circ}$ is met at $30 \%$ sc at the GP with $\sim 55 \mathrm{~nm}$ RMS contingency.

(b) $227 \mathrm{~nm}$ at $\psi=45^{\circ}$ is met at $50 \% \mathrm{sc}$ at $30^{\circ}$ GL with $\sim 83 \mathrm{~nm}$ RMS contingency.

Full scientific assessment of off-zenith performance versus sky coverage is underway, but preliminary analysis on a set of 75 specific target fields covering 5 different field types (extragalactic, nearby galaxies, central black holes, massive young star clusters, and yellow super giants) indicate that only a few percent of these targets are not expected to meet the extrapolated ORD WFE requirement at their culminating elevation angle (minimum zenith angle) with $50 \%$ or better probability under median Mauna Kea turbulence conditions. Fig.5 illustrates the distribtion of these target fields as well as the estimated region with $<50 \%$ sc.

\section{Acknowledgments}

The authors gratefully acknowledge the support of the TMT partner institutions. They are the Association of Canadian Universities for Research in Astronomy (ACURA), the California Institute of Technology and the University of California. This work was supported as well by the Gordon and Betty Moore Foundation, the Canada Foundation for Innovation, the Ontario Ministry of Research and Innovation, the National Research 


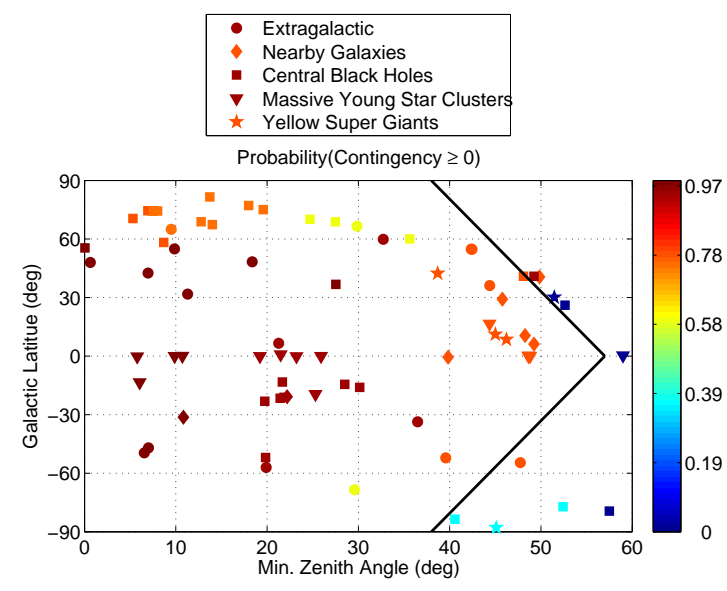

Figure 5. Map of 75 potential science target fields as well as estimated region with $<50 \%$ sc.

Council of Canada, the Natural Sciences and Engineering Research Council of Canada, the British Columbia Knowledge Development Fund, the Association of Universities for Research in Astronomy (AURA) and the U.S. National Science Foundation.

\section{REFERENCES}

1. B.Ellerbroek, S.Adkins, D.Andersen, J.Atwood, S.Browne, C.Boyer, P.Byrnes, K.Caputa, R.Cousty, D.Erikson, J.Fitzsimmons, F.Gamache, L.Gilles, G.Herriot, P.Hickson, O.Lardière, P.Morin, J.Pazder, T.Pfrommer, D.Quinn, V.Reshetov, S.Roberts, J.-C.Sinquin, M.Schoeck, M.Smith, G. Tyler, J.Vaughn, J.P.Véran, C.Vogel, L.Wang and I.Wevers, "First light adaptive optics systems and components for the Thirty Meter Telescope", Proc. Soc. Photo-Opt. Instrum. Eng. 7736-3 (2010).

2. G.Herriot, D.Andersen, J.Atwood, C.Boyer, P.Byrnes, R.Conan, B.Ellerbroek, J. Fitzsimmons, L.Gilles, P.Hickson, A.Hill, K.Jackson, O.Lardière, T.Pfrommer, J.-P.Véran, L.Wang and I.Wevers, "NFIRAOS: facility adaptive optics system for the TMT", Proc. Soc. Photo-Opt. Instrum. Eng. 7736-9 (2010).

3. J.Larkin, A.Moore, E.Barton,B.Bauman, K.Bui, J.Canfield, D.Crampton, A.Delacroix, M.Fletcher, D.Hale, D.Loop, C.Niehaus, A.Phillips, V.Reshetov, L.Simard, R.Smith, R.Suzuki, T.Usuda and S.Wright, "The infrared imaging spectrograph (IRIS) for TMT: instrument overview", Proc. Soc. Photo-Opt. Instrum. Eng. 7735-79 (2010).

4. B.Mobasher, R.Weber, N.Konidaris, D.Crampton, L.Simard, "An infrared multi-object spectrograph (IRMS) with adaptive optics on the TMT: overview and science case", Proc. Soc. Photo-Opt. Instrum. Eng. 7735-211 (2010).

5. L.Gilles and B.L.Ellerbroek, "Split atmospheric tomography using laser and natural guide stars", J. Opt. Soc. Am. A 25, 2427-2435 (2008).

6. L.Wang, B.Ellerbroek and J.P.Véran, "High fidelity sky coverage analysis via time domain adaptive optics simulations", Appl. Opt. 48, 5076-5087 (2009).

7. B.Ellerbroek, "Evaluating and optimizing control algorithms for combined LGS/NGS MCAO systems". Appendix E of the conceptual design review documents for MCAO for Gemini South, Gemini Observatory (2002). Available online at http://www.gemini.edu/sciops/instruments/adaptive-optics/.

8. Kalnay et al., "The NCEP/NCAR 40-year reanalysis project", Bull. Amer. Soc. 77, 437-470 (1996).

9. S.Adkins, "Laser systems for laser guide star adaptive optics: status and perspectives", Proc. Soc. Photo-Opt. Instrum. Eng. 7736-64 (2010).

10. L.Gilles and B.L.Ellerbroek, "Constrained Matched Filtering for Extended Dynamic Range and Improved Noise Rejection For Shack-Hartmann Wavefront Sensing", Opt. Lett. 33 (2008).

11. T.Pfrommer and P.Hickson, "High-resolution mesospheric sodium observations for extremely large telescopes", Proc. Soc. Photo-Opt. Instrum. Eng. 7736-71 (2010).

12. L.Wang, A.Otarola and B.Ellerbroek, "Impact of laser guide star fratricide on the TMT MCAO system", Proc. Soc. Photo-Opt. Instrum. Eng. 7736-16 (2010). 